comorbidity, ADHD and Childhood Bipolar Disorder, the sooner the child is on appropriate medications, the better. When just the surface diagnosis of ADHD is medicated, the outcome is often problematic. There may be a poor response to treatment and a higher rate of suicide.

133

\section{Toxic Psychosis: Follow-up After One Year of Treatment}

\section{Glen Oriaifo, $M D$}

Department of Psychiatry and Behavioral Medicine, Virginia Tech Carilion School of Medicine, Roanoke, VA

ABSTRACT: Introduction: Anti-NMDA (N-methyl-D-aspartate) receptor encephalitis often presents itself in psychiatric settings as first-break psychosis. I present a case of a 31-year-old female who returned to the clinic one year after being treated for NMDA receptor antibody encephalitis.

CASE REPORT: Ms. C is a 31 y/o female who returned to the clinic after one year of being discharged from the hospital for NMDA-receptor encephalitis with positive serological NR1 antibodies. She was initially admitted to our inpatient psychiatric facility for an unspecified psychotic disorder complicated with seizure-like episodes. She was given various psychotropic medications without any improvement. She was moderately responsive to olanzapine and lorazepam. Her condition gradually worsened; she stopped communicating and became mute. Neurology consultation prompted work-up for encephalitis and the probable diagnosis of NMDA receptor encephalitis. She was subsequently treated with steroids, IVIG and then intrathecal rituximab and bortezomib. In addition to these aforementioned medications, she underwent a prophylactic oophorectomy and 10 ECT treatments for life threatening catatonia. After three weeks of this regimen, Ms. C recovered completely and was discharged home.

DISCUSSION: This case adds to literature that suggests prompt diagnosis and management of NMDA receptor encephalitis significantly improves prognosis. Treatment should be initiated if the patient meets probable diagnostic criteria for NMDA receptor encephalitis. Similar to other cases in the literature, our patient's symptom of catatonia improved with ECT administration. During Ms. G one year follow-up, no evidence of psychotic symptoms were appreciated. Family members reported that she had returned to her baseline cognitive function.
134

Long-Term Deutetrabenazine Treatment Is Associated with Sustained Treatment Response in Tardive Dyskinesia: Results from an Open-Label Extension Study

Robert A. Hauser, MD, MBA'; Hadas Barkay, PhD ${ }^{2}$; Hubert H. Fernandez, $M^{3}$; Stewart A. Factor, DO ; Joohi Jimenez-Shahed, $\mathrm{MD}^{5}$; Nicholas Gross, $\mathrm{MS}^{6}$; Leslie Marinelli, $B S^{6}$; Mark Forrest Gordon, $M D^{6}$; Juha-Matti Savola, $M D, P h D^{7}$; and

Karen E. Anderson, $M D^{8}$

${ }^{1}$ University of South Florida Parkinson's Disease and Movement Disorders Center, Tampa, Florida, USA

${ }^{2}$ Teva Pharmaceuticals, Netanya, Israel

${ }^{3}$ Cleveland Clinic, Center for Neurological Restoration, Cleveland, Ohio, USA

${ }^{4}$ Emory University, Atlanta, Georgia, USA

${ }^{5}$ Baylor College of Medicine, Houston, Texas, USA

${ }^{6}$ Teva Pharmaceuticals, Frazer, Pennsylvania, USA

${ }^{7}$ Teva Pharmaceuticals International, Basel, Switzerland

${ }^{8}$ Georgetown University, Washington, District of

Columbia, USA

ABSTRACT: Background: In the 12-week ARM-TD and AIM-TD studies evaluating deutetrabenazine for the treatment of tardive dyskinesia (TD), the percentage of patients achieving $\geq 50 \%$ response was higher in the deutetrabenazine-treated group than in the placebo group. These studies also showed low rates of overall adverse events (AEs) and discontinuations associated with deutetrabenazine. The current open-label study evaluated the long-term efficacy and safety of deutetrabenazine in patients with TD.

METHODS: Patients with TD who completed ARM-TD or AIM-TD could enroll in this open-label, single-arm extension study, titrating up over 6 weeks to a maximum total daily dose of deutetrabenazine $48 \mathrm{mg}$ /day on the basis of dyskinesia control and tolerability. The proportion of Abnormal Involuntary Movement Scale (AIMS; items 1-7) responders was assessed based on response rates for achieving $\geq 50 \%$ improvement from baseline in the open-label extension study. AlMS score was assessed by local site raters for this analysis.

RESULTS: 343 patients enrolled in the extension study. At Week $54(\mathrm{n}=249$; total daily dose [mean \pm standard error]: $38.6 \pm 0.66 \mathrm{mg}$ ), the mean percentage change from baseline in AIMS score was $-40 \% ; 48 \%$ of patients achieved a $\geq 50 \%$ response and $59 \%$ of those had already achieved a $\geq 50 \%$ response at Week 15 . Further, $34 \%$ of those who had not achieved a $\geq 50 \%$ response at Week 15 achieved a $\geq 50 \%$ response at Week 54 . At Week $106(\mathrm{n}=169$; total daily dose: $39.6 \pm 0.77 \mathrm{mg})$, the mean 
percentage change from baseline in AIMS score was $45 \% ; 55 \%$ of patients achieved a $\geq 50 \%$ response, $59 \%$ of those patients had already achieved a $\geq 50 \%$ response at Week 15 , and $41 \%$ of those who had not achieved a $\geq 50 \%$ response at Week 15 but who reached Week 106 achieved a $\geq 50 \%$ response. At Week $132(\mathrm{n}=109$; total daily dose: $39.7 \pm 0.97 \mathrm{mg}$ ), the mean percentage change from baseline in AIMS score was $-61 \% ; 55 \%$ of patients achieved a $\geq 50 \%$ response, $61 \%$ of those patients had already achieved a $\geq 50 \%$ response at Week 15 , and $43 \%$ of those who had not achieved a $\geq 50 \%$ response at Week 15 but who reached Week 132 achieved a $\geq 50 \%$ response. Completer analysis suggests that long-term efficacy was not due to dose increases over time. Treatment with deutetrabenazine was generally well tolerated. There were 623 patient-years of exposure through Week 158, and exposure-adjusted incidence rates (incidence/patientyears) of adverse events of special interest were 0.01 for akathisia and restlessness, 0.07 for somnolence and sedation, 0.04 for parkinsonism, and 0.05 for depression.

CONCLUSIONS: Patients who received long-term treatment with deutetrabenazine achieved response rates that were indicative of clinically meaningful long-term benefit. Results from this open-label trial suggest the possibility of increasing benefit over time with individual dose titration of deutetrabenazine.

Funding Acknowledgements: This study was funded by Teva Pharmaceuticals, Petach Tikva, Israel.

\section{5}

Evolution of a Study of Bilateral Prefrontal Transcranial Magnetic Stimulation (TMS) to Treat the Symptoms of Mild TBI (mTBI) and PTSD

Jared S Garland, B.S. ' ; Emilia-Marie Jaskot, B.S. ${ }^{2}$; Jerika Taylor, $M A^{3}$; Kiara Buccellato, $M A^{4}$; Navneet Atwal, M.D. ${ }^{5}$; and Paul F Pasquina, M.D. ${ }^{6}$

${ }^{1}$ Clinical Research Assistant, The Henry M Jackson Foundation for the Advancement of Military Medicine, Center for Rehabilitation Sciences Research at the Uniformed Services University of the Health Sciences, Walter Reed National Military Medical Center, Bethesda, MD

${ }^{2}$ Clinical Research Assistant, The Henry M Jackson Foundation for the Advancement of Military Medicine, Center for Rehabilitation Sciences Research at the Uniformed Services University of the Health Sciences, Walter Reed National Military Medical Center, Bethesda, MD

${ }^{3}$ Program Manager, The Henry M Jackson Foundation for the Advancement of Military Medicine, Center for Rehabilitation Sciences Research at the Uniformed Services University of the Health Sciences, Walter Reed National Military Medical Center, Bethesda, MD
${ }^{4}$ Clinical Research Coordinator: Clinical Research Assistant, The Henry M Jackson Foundation for the Advancement of Military Medicine, Center for Rehabilitation Sciences Research at the Uniformed Services University of the Health Sciences, Walter Reed National Military Medical Center, Bethesda, MD ${ }^{5}$ Psychiatrist, Department of Psychiatry, Walter Reed National Military Medical Center, Bethesda, MD ${ }^{6}$ Chair, Center for Rehabilitation Sciences Research at the Uniformed Services University of the Health Sciences; Chief, Department of Rehabilitation at Walter Reed National Military Medical Center, Bethesda, MD

DISCLAIMER: The views expressed in this abstract are those of the authors and do not reflect the official policy of the Department of Army/Navy/Air Force, Department of Defense, or U.S. Government.

Keywords: PTSD, mTBI, compliance, Repetitive Transcranial Magnetic Stimulation, Neuromodulation

BACKGROUND: Traumatic brain injuries (TBIs) have affected nearly 380,000 service members since 2000 . Comorbid posttraumatic stress disorder (PTSD) may result from and/or exacerbate sequelae of mild TBI (mTBI) and is suspected to affect up to $65 \%$ of service members with TBI. Conventional treatments for mTBI/PTSD symptoms have limited efficacy and are associated with undesirable side effects. Repetitive transcranial magnetic stimulation (rTMS) has shown promise in treating PTSD symptoms and been identified as a potential mTBI therapy, but is untested as a therapy for comorbid mTBI/PTSD.

METHODS: This double-blinded, prospective randomized, sham-controlled study consists of 30 treatment sessions 5 weeks of daily sessions followed by a two week taper of 3 and 2 sessions, respectively. Sessions consist of 3500 pulses administered to the left dorsolateral prefrontal cortex (dlPFG) at $10 \mathrm{~Hz}$ and 1500 pulses to the right dlPFG at $1 \mathrm{~Hz}$. Approximately 60-80 participants will be randomized to receive active or sham rTMS. Primary outcome measures are the Posttraumatic Checklist 5 and the Rivermead Post-Concussion Questionnaire.

RESULTS: The study is ongoing, and 26 participants have been recruited to date. All patients were formally diagnosed with mTBI and reported moderate to severe PTSD symptoms. Preliminary data show no participants have withdrawn due to intolerability or indicated intolerability, despite the presence of minor discomforts such as headache. The majority of participants have been able to rest quietly or sleep during sessions, indicating high tolerability. Reported pain levels are low, with average ratings of $2.84 / 10.00$ by week 2 . One limitation was a high dropout rate. 